\title{
The properties of $\bar{K}$ in the nuclear medium
}

\author{
A. Ramos \\ Departament d'Estructura i Constituents de la Matèria, Universitat de Barcelona, \\ Diagonal 647, 08028 Barcelona, Spain \\ E. Oset \\ Departamento de Física Teórica and IFIC, Centro Mixto Universidad de Valencia-CSIC, \\ 46100 Burjassot (Valencia), Spain
}

(November 6, 2018)

\begin{abstract}
The self-energy of the $K^{-}$meson in nuclear matter is calculated in a selfconsistent microscopic approach, using a $\bar{K} N$ interaction obtained from the lowest-order meson-baryon chiral Lagrangian. The effective $\bar{K} N$ interaction in the medium is derived by solving the coupled-channel Bethe-Salpeter equation including Pauli blocking on the nucleons, mean-field binding potentials for the baryons and the self-energy of the $\pi$ and $\bar{K}$ mesons. The incorporation of the self-consistent $\bar{K}$ self-energy in the description, in addition to the Pauli blocking effects, yields a weaker attractive in-medium $\bar{K} N$ interaction and a $\Lambda(1405)$ which dissolves faster with increasing matter density, as a result of the $\bar{K}$ spectral function being spread out over a wide range of energies. These effects are further magnified when the intermediate pions are dressed.
\end{abstract}

PACS: 12.38.Lg, 13.75.Jz, 14.20.Gk, 14.20.Jn, 14.40.Aq, 21.65.+f, 25.80.Nv

Keywords: $\bar{K} N$ interaction, Chiral Lagrangian, $\Lambda(1405)$ in nuclear matter, Effective kaon mass, Kaonic atoms.

\section{INTRODUCTION}

The properties of the kaons and antikaons in the nuclear medium have been the object of numerous investigations since the possibility of the existence of a kaon condensed phase in dense nuclear matter was pointed out [1]. The enhancement of the $K^{-}$yield in $\mathrm{Ni}+\mathrm{Ni}$ collisions measured recently by the KaoS collaboration at GSI [2] can be explained by assuming the $K^{-}$meson to feel a strong attraction in the medium [3, [1]. Kaonic atom data, a compilation of which is given in Ref. [5], also favor an attractive $K^{-}$nucleus interaction.

Most of the recent theoretical works start from chiral Lagrangians that reproduce the free space scattering properties which, in the case of $K^{-} p$ scattering, are dominated by the presence of an isospin zero resonance, the $\Lambda(1405)$. It is precisely the influence of 
this resonance what makes the $K^{-} p$ interaction repulsive at threshold, while the lowest order Born s-wave amplitude from the chiral Lagrangian (the Tomozawa-Weinberg term) is attractive. Evidently, one cannot expect chiral perturbation theory to work close to a resonance. This is why all works studying the free space $K^{-} N$ interaction from the chiral Lagrangian either introduce the $\Lambda(1405)$ as an elementary field [6] or generate it dynamically through the Lippmann-Schwinger [7] or the Bethe-Salpeter [B] equations. These latter approaches allow for a microscopic incorporation of the medium effects on the the $\bar{K} N$ interaction [9 12]. For instance, Pauli blocking on the intermediate nucleon states, makes the $\bar{K} N$ interaction density dependent and this, in turn, modifies the $K^{-}$properties from those in free space. These medium modifications were already included long time ago in the context of Brueckner-type many body theory [13] to obtain the kaon-nucleus optical potential for kaonic atoms. The medium properties of kaons and antikaons have also been obtained from mean field theories, built within the framework of chiral Lagrangians [4, 14], based on the relativistic Walecka-type model extended to incorporate strangeness in the form of hyperons or kaons [15], or using explicitly quark degrees of freedom [16].

All the different approaches agree qualitatively in establishing that, in the medium, the $K^{+}$feels a moderate repulsion and the $K^{-}$a strong attraction. How large is this attraction is still somewhat controversial. Recent phenomenological approaches [0] based on fits to kaonic atom data find a $K^{-}$-nucleus potential of the order of $-200 \pm 20 \mathrm{MeV}$ in the nuclear center. However, no calculation that starts from the bare $K^{-} N$ interaction predicts such an attraction, the values ranging from -140 to $-75 \mathrm{MeV}$. Hopefully, heavy-ion reactions, that are sensitive to higher density regions, will help in elucidating these discrepancies. At the same time, it is necessary to develop theories that treat the intricacies associated to the mutual interaction between all the hadrons in the medium as accurately as possible.

In the present work we perform a microscopic study of the the $K^{-}$properties in nuclear matter by incorporating the medium modifications on the $\bar{K} N$ amplitude using the model of Ref. [8], which was shown to reproduce the $K^{-} p$ scattering observables very satisfactorily. As mentioned above, one source of density dependence is the Pauli blocking on the nucleon states. This makes the $\bar{K} N$ interaction attractive and, on the other hand, shifts the resonance to higher energies [9,11]. However, it was shown recently [12 that a self-consistent calculation of the $K^{-}$self-energy leaves the position of the resonance unchanged, due to a compensation of the repulsive $\Lambda(1405)$ shift with the attraction felt by the $K^{-}$meson. The importance of these medium effects makes it interesting to investigate other medium modifications of the particles participating in building up the $\bar{K} N$ interaction. This is the aim of the present work. To this end, we include, in addition, the dressing of the pions in the $\pi \Lambda, \pi \Sigma$ intermediate states, which couple strongly to the $\bar{K} N$ state. This is done through a pion self-energy that contains the effect of one- and two-nucleon absorption, conveniently modified to include the effect of nuclear short-range correlations. The medium effects on the nucleons and hyperons is considered via density-dependent mean-field binding potentials.

After reviewing the free-space formalism in Sect. II, we describe, in Sect. III, the appropiate modifications needed to incorporate the medium effects on the $\bar{K} N$ amplitude. The details of the kaon and pion self-energies are given in Sect. IV. Our results are discussed in Sect. D, where various approximations are compared. Finally, Sect. VI summarizes our main results and conclusions. 


\section{II. $\bar{K} N$ AMPLITUDES IN FREE SPACE}

In this section we review the formalism used in Ref. [8] for describing $\bar{K} N$ scattering in free space. The effective chiral Lagrangian formalism has been very successful in explaining the properties of meson-meson interaction at low energies [17,18], as well as those of the meson-baryon systems [19,20] when the interaction is weak, as in the case of the s-wave $\pi N$ and $K^{+} N$ interaction. However, the $\bar{K} N$ system couples strongly to many other channels and generates a resonance below threshold, the $\Lambda(1405)$. In this case the standard chiral scheme, an expansion in powers of the typical momenta involved in the process, fails to be an appropriate approach.

In Ref. 8] a non perturbative scheme, consisting of solving a set of coupled-channel Bethe-Salpeter equations using the lowest order chiral Lagrangian in S-wave, was shown to reproduce the low energy $K^{-} p$ scattering data very satisfactorily with only one-parameter, the cut-off used to renormalize the loop integrals. A reinterpretation of these equations to the light of the inverse amplitude method can be seen in Ref. [21], where it is shown that the effect of higher order chiral Lagrangians can be reabsorbed with the choice of a suitable cut-off in some cases. The model of Ref. [8] follows closely that of Ref. [0], where the success of the non-perturbative approach using the chiral Lagrangians was first shown, but includes the complete set of $0^{-}$meson and $1 / 2^{+}$baryon octet states in the basis space of coupled channels. The use of this complete set, apart from providing the right $\mathrm{SU}(3)$ symmetry in the limit of equal baryon masses and equal meson masses, was found to be essential for reproducing the experimental branching ratios at threshold with only the lowest order Lagrangian.

The lowest order chiral Lagrangian, coupling the octet of pseudoscalar mesons to the octet of $1 / 2^{+}$baryons, is

$$
\begin{aligned}
L_{1}^{(B)}= & \left\langle\bar{B} i \gamma^{\mu} \nabla_{\mu} B\right\rangle-M_{B}\langle\bar{B} B\rangle \\
+ & \frac{1}{2} D\left\langle\bar{B} \gamma^{\mu} \gamma_{5}\left\{u_{\mu}, B\right\}\right\rangle+\frac{1}{2} F\left\langle\bar{B} \gamma^{\mu} \gamma_{5}\left[u_{\mu}, B\right]\right\rangle,
\end{aligned}
$$

where the symbol \langle\rangle denotes the trace of $\mathrm{SU}(3)$ matrices and

$$
\begin{aligned}
& \nabla_{\mu} B=\partial_{\mu} B+\left[\Gamma_{\mu}, B\right] \\
& \Gamma_{\mu}=\frac{1}{2}\left(u^{\dagger} \partial_{\mu} u+u \partial_{\mu} u^{\dagger}\right) \\
& U=u^{2}=\exp (i \sqrt{2} \Phi / f) \\
& u_{\mu}=i u^{\dagger} \partial_{\mu} U u^{\dagger} .
\end{aligned}
$$

The SU(3) matrices for the mesons and the baryons are the following

$$
\begin{gathered}
\Phi=\left(\begin{array}{ccc}
\frac{1}{\sqrt{2}} \pi^{0}+\frac{1}{\sqrt{6}} \eta & \pi^{+} & K^{+} \\
\pi^{-} & -\frac{1}{\sqrt{2}} \pi^{0}+\frac{1}{\sqrt{6}} \eta & K^{0} \\
K^{-} & \bar{K}^{0} & -\frac{2}{\sqrt{6}} \eta
\end{array}\right), \\
B=\left(\begin{array}{ccc}
\frac{1}{\sqrt{2}} \Sigma^{0}+\frac{1}{\sqrt{6}} \Lambda & \Sigma^{+} & p \\
\Sigma^{-} & -\frac{1}{\sqrt{2}} \Sigma^{0}+\frac{1}{\sqrt{6}} \Lambda & n \\
\Xi^{-} & \Xi^{0} & -\frac{2}{\sqrt{6}} \Lambda
\end{array}\right) .
\end{gathered}
$$


At lowest order in momentum the interaction Lagrangian reduces to

$$
L_{1}^{(B)}=\left\langle\bar{B} i \gamma^{\mu} \frac{1}{4 f^{2}}\left[\left(\Phi \partial_{\mu} \Phi-\partial_{\mu} \Phi \Phi\right) B-B\left(\Phi \partial_{\mu} \Phi-\partial_{\mu} \Phi \Phi\right)\right]\right\rangle .
$$

The coupled channel formalism requires to evaluate the transition amplitudes between the different channels that can be built from the meson and baryon octets. For $K^{-} p$ scattering there are 10 channels, namely $K^{-} p, \bar{K}^{0} n, \pi^{0} \Lambda, \pi^{0} \Sigma^{0}, \pi^{+} \Sigma^{-}, \pi^{-} \Sigma^{+}, \eta \Lambda, \eta \Sigma^{0}, K^{+} \Xi^{-}$and $K^{0} \Xi^{0}$. In the case of $K^{-} n$ scattering the coupled channels are: $K^{-} n, \pi^{0} \Sigma^{-}, \pi^{-} \Sigma^{0}, \pi^{-} \Lambda$, $\eta \Sigma^{-}$and $K^{0} \Xi^{-}$. These amplitudes have the form

$$
V_{i j}=-C_{i j} \frac{1}{4 f^{2}} \bar{u}\left(p_{i}\right) \gamma^{\mu} u\left(p_{j}\right)\left(k_{i \mu}+k_{j \mu}\right),
$$

where $p_{j}, p_{i}\left(k_{j}, k_{i}\right)$ are the initial, final momenta of the baryons (mesons). The explicit values of the coefficients $C_{i j}$ can be found in Ref. [8]. At low energies we can neglect the spatial components and Eq. (6) simplifies to

$$
V_{i j}=-C_{i j} \frac{1}{4 f^{2}}\left(k_{j}^{0}+k_{i}^{0}\right) .
$$

The coupled-channel Bethe-Salpeter equations in the center of mass frame read

$$
T_{i j}=V_{i j}+\overline{V_{i l} G_{l} T_{l j}},
$$

where the indices $i, l, j$ run over all possible channels and

$$
\overline{V_{i l} G_{l} T_{l j}}=i \int \frac{d^{4} q}{(2 \pi)^{4}} \frac{M_{l}}{E_{l}(-\vec{q})} \frac{V_{i l}\left(k_{i}, q\right) T_{l j}\left(q, k_{j}\right)}{k^{0}+p^{0}-q^{0}-E_{l}(-\vec{q})+i \epsilon} \frac{1}{q^{2}-m_{l}^{2}+i \epsilon},
$$

with $M_{l}, E_{l}$ and $m_{l}$ being, respectively, the baryon mass, baryon energy and meson mass in the intermediate state.

Although Eq. (9) requires the half-off-shell amplitudes, it was shown in Ref. [8] that the off-shell part goes into renormalization of coupling constants. Therefore, one can factorize the remainig on-shell components of $V$ and $T$ outside the integral of Eq. (9) reducing the integral equation to a set of algebraic equations. The loop integral reads

$$
\begin{aligned}
G_{l}(\sqrt{s}) & =i \int \frac{d^{4} q}{(2 \pi)^{4}} \frac{M_{l}}{E_{l}(-\vec{q})} \frac{1}{\sqrt{s}-q^{0}-E_{l}(-\vec{q})+i \epsilon} \frac{1}{q^{2}-m_{l}^{2}+i \epsilon} \\
& =\int_{|\vec{q}|<q_{\max }} \frac{d^{3} q}{(2 \pi)^{3}} \frac{1}{2 \omega_{l}(\vec{q})} \frac{M_{l}}{E_{l}(-\vec{q})} \frac{1}{\sqrt{s}-\omega_{l}(\vec{q})-E_{l}(-\vec{q})+i \epsilon}
\end{aligned}
$$

with $\sqrt{s}=p^{0}+k^{0}$.

The model discussed here depends on the loop regularization cut-off, whose value $q_{\max }=$ $630 \mathrm{MeV}$ was chosen to reproduce the $K^{-} p$ scattering branching ratios at threshold [8]. The weak decay constant is $f=1.15 f_{\pi}$, a value lying in between the pion and kaon ones that

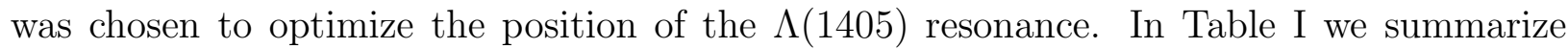
the predictions of the model for several scattering observables at threshold. The scattering cross sections, which are not used in the fit, were shown to be in good agreement with the low energy data [8] and the model can safely be used up to $500 \mathrm{MeV} / \mathrm{c} K^{-}$lab momentum [26] up to some punctual discrepancy in the small $K^{-} p \rightarrow \bar{K}^{0} n$ cross section around 400 $\mathrm{MeV} / \mathrm{c}$, where the D-wave $\Lambda(1520)$ resonance, not accounted for in the theory, shows up in the data. 


\section{III. $\bar{K}$ IN THE NUCLEAR MEDIUM}

The dynamics of the $\bar{K} N$ interaction at low energies is dominated by the $\Lambda(1405)$ resonance, which in the approaches of Refs. [7,8 is interpreted as an isospin $I=0$ quasi-bound $K^{-} p$ state. The $\Lambda(1405)$ resonance is slightly below the $K^{-} p$ threshold and leads to a repulsive $K^{-} p$ amplitude. Although the isospin $I=1 K^{-} n$ channel is attractive [24], the $K^{-} p, K^{-} n$ averaged amplitude, $\bar{T}$, is still repulsive and, according to the low energy theorem, the $K^{-}$self-energy $(\bar{T} \rho)$ should also be repulsive. However, kaonic atom data suggest that, even at a small fraction of the normal nuclear matter density $\rho_{0}$, the $K^{-}$feels a strongly attractive potential. This implies a rapid transition from a repulsive $\bar{K} N$ interaction to an attractive one as density increases and a microscopic study of the $K^{-}$properties in the medium cannot be done in terms of the simple $\bar{T} \rho$ or impulse approximation. It is therefore necessary to consider the density dependence of the in-medium $\bar{K} N$ interaction, $T_{\text {eff }}(\rho)$.

One source of density dependence comes from the Pauli principle, which prevents the scattering to intermediate nucleon states below the Fermi momentum, $p_{F}$. To incorporate this effect in the channels having an intermediate nucleon state, one must replace the free nucleon propagator by the in-medium one in the loop integral of Eq. (10). The evaluation of the $K^{-}$self-energy will require the knowledge of the $K^{-} N T$-matrix at momenta $P=p_{N}+p_{K}$ in the nuclear lab frame, where $p_{N}$ is a nucleon momentum from the Fermi sea and $p_{K}$ is the momentum of the $K^{-}$. The loop integral of Eq. (10) used in the description of the $K^{-} N$ scattering data is evaluated and regularized by means of a cut-off in the center-ofmass frame. In order to be able to use the same formalism we boost the $K^{-} N$ system to its center-of-mass frame and then evaluate the loop function. However, since Pauli blocking is most easily implemented in the lab frame, we express the occupation number in terms of the momentum of the intermediate nucleon in the laboratory, $\vec{q}_{\text {lab }}$. Hence, we have

$$
\begin{aligned}
G_{l}\left(P^{0}, \vec{P}, \rho\right) & =i \int \frac{d^{4} q}{(2 \pi)^{4}} \frac{M_{l}}{E_{l}(-\vec{q})}\left\{\frac{1-n\left(\vec{q}_{\text {lab }}\right)}{\sqrt{s}-q^{0}-E_{l}(-\vec{q})+i \epsilon}+\frac{n\left(\vec{q}_{\text {lab }}\right)}{\sqrt{s}-q^{0}-E_{l}(-\vec{q})-i \epsilon}\right\} \\
& \times \frac{1}{q^{2}-m_{l}^{2}+i \epsilon},
\end{aligned}
$$

where $\left(P_{0}, \vec{P}\right)$ is the total four-momentum in the lab frame, $s=\left(P^{0}\right)^{2}-\vec{P}^{2}$ and

$$
\vec{q}_{\mathrm{lab}}=\left[-\left(\frac{P^{0}}{\sqrt{s}}-1\right) \frac{\vec{P} \vec{q}}{|\vec{P}|^{2}}+\frac{\sqrt{s}-q^{0}}{\sqrt{s}}\right] \vec{P}-\vec{q}
$$

is the nucleon momentum in the lab frame corresponding to a momentum $-\vec{q}$ in the centerof-mass frame.

As one can see from Eq. (11), the Pauli blocking corrections are only operative at the nucleon pole. Indeed, by collecting the terms proportional to $n\left(\vec{q}_{\text {lab }}\right)$ in the nucleon propagator one finds $2 \pi i n\left(\vec{q}_{\text {lab }}\right) \delta\left(\sqrt{s}-q^{0}-E_{l}(-\vec{q})\right)$, which sets the value of $q^{0}$ to be used in Eq. (12). This allows one to perform the integration over $q^{0}$ in Eq. (11) analytically resulting in

$$
G_{l}\left(P^{0}, \vec{P}, \rho\right)=\int_{|\vec{q}|<q_{\max }} \frac{d^{3} q}{(2 \pi)^{3}} \frac{1}{2 \omega_{l}(\vec{q})} \frac{M_{l}}{E_{l}(-\vec{q})}\left\{\frac{1-n\left(\vec{q}_{\text {lab }}\right)}{\sqrt{s}-\omega_{l}(\vec{q})-E_{l}(-\vec{q})+i \epsilon}\right.
$$




$$
\left.+\frac{n\left(\vec{q}_{\text {lab }}\right)}{\sqrt{s}+\omega_{l}(\vec{q})-E_{l}(-\vec{q})-i \epsilon}\right\},
$$

In practice, we have checked that the value $\sqrt{s}-q^{0}=E(-\vec{q})$ to be used in Eq. (12) can be replaced, with negligible changes, by the energy of the nucleon when both the meson and the baryon are placed on-shell

$$
\sqrt{s}-q^{0}=\frac{s+M_{l}^{2}-m_{l}^{2}}{2 \sqrt{s}}
$$

The second term on the right hand side of Eq. (13), related to the hole part of the nucleon propagator, is purely real for the kinematical conditions studied in this work, since one always finds $\sqrt{s}+\omega(\vec{q})-E_{l}(-\vec{q})>0$.

All works studying the Pauli blocking effects on the in-medium $\bar{K} N$ interaction [9] 12$]$ have neglected the hole term of Eq. (13), a simplification that is well justified for low densities since the phase space for holes is much reduced with respect to that for particles. However, this effect can become important if one wants to find the properties of the $\bar{K}$ at high densities as those achieved in heavy ion reactions or neutron stars.

Another source of density dependence is related to the fact that all the mesons and baryons participating in the intermediate loops interact with the nucleons of the Fermi sea and, as a consequence, feel a binding potential which changes the threshold energy of the different channels.

The binding effects on the baryons are taken within a mean-field approach consisting in adding, to the single particle energies in Eqs. (10) and (13), a momentum-independent potential of the type $U=U_{0} \rho / \rho_{0}$, where $\rho_{0}=0.17 \mathrm{fm}^{-3}$ is normal nuclear matter density. In the case of nucleons a reasonable value is $U_{0}=-50 \mathrm{MeV}$ as implied by numerous nuclear structure data. On the other hand, since the experimental binding energy of a $\Lambda$ particle in hypernuclei extrapolates to about $30 \mathrm{MeV}$ for the very heavy systems [27], we take the potential depth of the $\Lambda$ to be $U_{0}=-30 \mathrm{MeV}$. Finally, although there is very little information on the $\Sigma$ single particle states, the recent measurement of the $\Sigma$ binding energy of about $4 \mathrm{MeV}$ in ${ }_{\Sigma}^{4} \mathrm{He}$ [28] indicates that the $\Sigma$ can certainly be bound in the nucleus. Moreover, analysis of $\Sigma$-atom data by Batty et al [29] and from Ref. [30] found the data to be compatible with a potential of the type $U_{0} \rho / \rho_{0}$ with $U_{0}$ about -25 to $-30 \mathrm{MeV}$. Based on this evidence, we take $U_{0}=-30 \mathrm{MeV}$ for the $\Sigma$ potential.

The nuclear medium effects on the mesons will be included through the corresponding self-energy. We will only consider the dressing of the $\bar{K}$ and $\pi$ mesons since the $\eta$ and $K$ mesons appear in intermediate states that lie quite far above the $K^{-} p$ threshold. In the next section, we show how the $\bar{K}$ and $\pi$ self-energies are constructed. Here, we will focus on how the dressed meson propagator is incorporated into the scheme. To this end, we write the meson propagator $(i=\bar{K}, \pi)$

$$
D_{i}\left(q^{0}, \vec{q}, \rho\right)=\frac{1}{\left(q^{0}\right)^{2}-\vec{q}^{2}-m_{i}^{2}-\prod_{i}\left(q^{0}, \vec{q}, \rho\right)}
$$

in the Lehmann representation

$$
D_{i}\left(q^{0}, \vec{q}, \rho\right)=-\frac{1}{\pi} \int_{0}^{\infty} d \omega \frac{\operatorname{Im} D_{i}(\omega, \vec{q}, \rho)}{q^{0}-\omega+i \epsilon}+\frac{1}{\pi} \int_{0}^{\infty} d \omega \frac{\operatorname{Im} D_{i}(\omega, \vec{q}, \rho)}{q^{0}+\omega-i \epsilon}
$$




$$
=\int_{0}^{\infty} d \omega 2 \omega \frac{S_{i}(\omega, \vec{q}, \rho)}{\left(q^{0}\right)^{2}-\omega^{2}+i \epsilon},
$$

where the spectral density, defined as

$$
S_{i}(\omega, \vec{q}, \rho)=-\frac{1}{\pi} \operatorname{Im} D_{i}(\omega, \vec{q}, \rho)=-\frac{1}{\pi} \frac{\operatorname{Im}_{i}(\omega, \vec{q}, \rho)}{\left|\omega^{2}-\vec{q}^{2}-m_{i}^{2}-\Pi_{i}(\omega, \vec{q}, \rho)\right|^{2}},
$$

has been introduced.

The free meson propagator in Eq. (13) must now be replaced by the dressed one written as in Eq. (16). While the $q^{0}$ integral proceeds as before, there now appears an additional integration over the variable $\omega$ running over all possible excited states to which a meson of momentum $\vec{q}$ can couple. The new meson-baryon loop integral is $(l=\bar{K}, \pi)$

$$
\begin{aligned}
G_{l}\left(P^{0}, \vec{P}, \rho\right) & =\int_{|\vec{q}|<q_{\max }} \frac{d^{3} q}{(2 \pi)^{3}} \frac{M_{l}}{E_{l}(-\vec{q})} \int_{0}^{\infty} d \omega S_{l}(\omega, \vec{q}, \rho) \\
& \times\left\{\frac{1-n\left(\vec{q}_{\text {lab }}\right)}{\sqrt{s}-\omega-E_{l}(-\vec{q})+i \epsilon}+\frac{n\left(\vec{q}_{\text {lab }}\right)}{\sqrt{s}+\omega-E_{l}(-\vec{q})}\right\},
\end{aligned}
$$

where $n\left(\vec{q}_{\text {lab }}\right)=0$ for the intermediate states involving hyperons $(\pi \Lambda$ and $\pi \Sigma)$.

If only Pauli blocking effects are considered, the effective interaction $T_{\text {eff }}\left(P^{0}, \vec{P}, \rho\right)$ is obtained by solving the coupled-channel Bethe-Salpeter equation using the meson-baryon propagator of Eq. (13) for the intermediate loops involving nucleons and that of Eq. (10) otherwise. If one also incorporates the dressing of the mesons, one must solve the BetheSalpeter equation using the meson-baryon propagator of Eq. (18).

\section{MESON SELF-ENERGIES}

The model discussed in Sect. II for the $\bar{K} N$ interaction gives rise to a s-wave $\bar{K}$ selfenergy $\left(\bar{K}=K^{-}\right.$or $\left.\bar{K}^{0}\right)$

$$
\Pi_{\bar{K}}^{s}\left(q^{0}, \vec{q}, \rho\right)=2 \int \frac{d^{3} p}{(2 \pi)^{3}} n(\vec{p})\left[T_{\text {eff }}^{\bar{K} p}\left(P^{0}, \vec{P}, \rho\right)+T_{\text {eff }}^{\bar{K} n}\left(P^{0}, \vec{P}, \rho\right)\right],
$$

which is obtained by summing the in-medium $\bar{K} N$ interaction, $T_{\text {eff }}^{\alpha}(\alpha=\bar{K} p, \bar{K} n)$, over the nucleons in the Fermi sea. The values $\left(q^{0}, \vec{q}\right)$ stand now for the energy and momentum of the $\bar{K}$ in the lab frame. Note that a self-consistent approach is required since one calculates the $\bar{K}$ self-energy from the effective interaction $T_{\text {eff }}$ which uses $\bar{K}$ propagators which themselves include the self-energy being calculated.

We also include a p-wave contribution to the $\bar{K}$ self-energy coming from the coupling of the $\bar{K}$ meson to hyperon particle-nucleon hole $\left(Y N^{-1}\right)$ excitations. The $K^{-}$meson can couple to $p \Lambda, p \Sigma^{0}$ or $n \Sigma^{-}$and the $\bar{K}^{0}$ to $n \Lambda, n \Sigma^{0}$ or $p \Sigma^{-}$. The vertices $M B B^{\prime}$ are easily derived from the $D$ and $F$ terms of Eq. (1), expanding $U$ up to one meson field. Using a non-relativistic reduction of the $\gamma^{\mu} \gamma^{5}$ matrix, one finds

$$
-i t_{M B B^{\prime}}=D_{M B B^{\prime}} \vec{\sigma} \vec{q}=\left(\alpha_{M B B^{\prime}} \frac{D+F}{2 f_{\pi}}+\beta_{M B B^{\prime}} \frac{D-F}{2 f_{\pi}}\right) \vec{\sigma} \vec{q},
$$


where $\vec{q}$ is the kaon momentum. We take $D+F=g_{A}=1.257$ and $D-F=0.33$. The coefficients $\alpha_{M B B^{\prime}}$ and $\beta_{M B B^{\prime}}$ are listed in Table [I].

The p-wave self-energy for the $K^{-}$meson reads

$$
\Pi_{K^{-}}\left(q^{0}, \vec{q}, \rho_{n}, \rho_{p}\right)=\vec{q}^{2}\left(D_{K^{-} p \Lambda}^{2} U_{\Lambda}\left(q^{0}, \vec{q}, \rho_{p}\right)+D_{K^{-} p \Sigma^{0}}^{2} U_{\Sigma}\left(q^{0}, \vec{q}, \rho_{p}\right)+D_{K^{-} n \Sigma^{-}}^{2} U_{\Sigma}\left(q^{0}, \vec{q}, \rho_{n}\right)\right)
$$

where $U_{Y}\left(q^{0}, \vec{q}, \rho_{i}\right)$ is the generalized Lindhard function for different particle and hole masses in a medium of density $\rho_{i}$, with $\rho_{i}=\rho_{n}$ or $\rho_{p}$. As shown in the appendix of Ref. [30], it reads

$$
U_{Y}\left(q^{0}, \vec{q}, \rho_{i}\right)=\operatorname{Re} U_{Y}\left(q^{0}, \vec{q}, \rho_{i}\right)+i \operatorname{Im} U_{Y}\left(q^{0}, \vec{q}, \rho_{i}\right)
$$

where

$$
\begin{aligned}
& \operatorname{Re} U_{Y}\left(q^{0}, \vec{q}, \rho_{i}\right)=\frac{3}{2} \rho_{i} \frac{M_{Y}}{q p_{F}^{i}}\left\{z+\frac{1}{2}\left(1-z^{2}\right) \ln \frac{|z+1|}{|z-1|}\right\} \\
& \operatorname{Im} U_{Y}\left(q^{0}, \vec{q}, \rho_{i}\right)=-\pi \frac{3}{4} \rho_{i} \frac{M_{Y}}{q p_{F}^{i}}\left\{\left(1-z^{2}\right) \theta(1-|z|)\right\}
\end{aligned}
$$

and

$$
z=\left(q^{0}-\frac{q^{2}}{2 M_{Y}}-\left(M_{Y}-M\right)\right) \frac{M_{Y}}{q p_{F}^{i}}
$$

An analogous expression is found for the $\bar{K}^{0}$ meson. In symmetric nuclear matter, as is considered here, both self-energies are the same and reduce to

$$
\Pi_{\bar{K}}^{p}\left(q^{0}, \vec{q}, \rho\right)=\frac{1}{2}\left(\frac{g_{N \Lambda K}}{2 M}\right)^{2} \vec{q}^{2} U_{\Lambda}\left(q^{0}, \vec{q}, \rho\right)+\frac{3}{2}\left(\frac{g_{N \Sigma K}}{2 M}\right)^{2} \vec{q}^{2} U_{\Sigma}\left(q^{0}, \vec{q}, \rho\right)
$$

where $\frac{g_{N \Lambda K}}{2 M}=D_{K^{-} p \Lambda}=D_{\bar{K}^{0} n \Lambda}$ and $\frac{g_{N \Sigma K}}{2 M}=D_{K^{-} p \Sigma^{0}}$ are the generic $K N \Lambda$ and $K N \Sigma$ coupling constants, respectively. This p-wave contribution to the $\bar{K}$ self-energy can become important for large momentum values.

The solution of the new $K^{-}$dispersion relation

$$
\omega^{2}=\vec{q}^{2}+m_{K}^{2}+\operatorname{Re} \Pi_{\bar{K}}(\omega, \vec{q}, \rho)
$$

with $\Pi_{\bar{K}}(\omega, \vec{q}, \rho)=\Pi_{\bar{K}}^{s}(\omega, \vec{q}, \rho)+\Pi_{\bar{K}}^{p}(\omega, \vec{q}, \rho)$, determines the effective mass, $m_{K}^{*}=\operatorname{Re} \omega(\vec{q}=$ $0)$, and decay width, $\Gamma=-2 \operatorname{Im} \omega(\vec{q}=0)$, of the $K^{-}$meson in the medium.

For the pion self-energy we take that of Ref. [32] which consists of a small momentum independent s-wave part, $\Pi_{\pi}^{s}(\rho)$, plus a p-wave part, $\Pi_{\pi}^{p}\left(q^{0}, \vec{q}, \rho\right)$. The latter, constructed by allowing the pion to couple to particle-hole $(1 p 1 h), \Delta$-hole $(\Delta h)$ and two-particle-hole $(2 p 2 h)$ excitations, can be written as

$$
\Pi_{\pi}^{p}\left(q^{0}, \vec{q}, \rho\right)=\left(\frac{g_{\pi N N}}{2 M}\right)^{2} \vec{q}^{2} F^{2}(q)\left(U_{N}\left(q^{0}, \vec{q}, \rho\right)+U_{\Delta}\left(q^{0}, \vec{q}, \rho\right)+U_{2 p 2 h}\left(q^{0}, \vec{q}, \rho\right)\right)
$$


where $F(q)$ is a monopole form factor with cut-off $\Lambda_{\pi}=1.2 \mathrm{GeV}, U_{N}\left(U_{\Delta}\right)$ is the Lindhard function for $1 p 1 h(\Delta h)$ excitations and $U_{2 p 2 h}$ is a phenomenological function accounting for

$2 p 2 h$ excitations. The $U_{2 p 2 h}$ contribution at $\left(q^{0}, \vec{q}, \rho\right)=\left(m_{\pi}, \overrightarrow{0}, 0.75 \rho_{0}\right)$ was obtained from fits to pionic atom data [31]. The extension to kinematical regions away from pionic atoms was done by multiplying the imaginary part by the available phase space of $2 p 2 h$ states for the given $q^{0}, \vec{q}$ values at density $\rho$. The explicit expression can be found in Ref. [32].

The pion self-energy is further modified to include the effect of nuclear short-range correlations. This is accomplished by replacing the self-energy of Eq. (27) by

$$
\Pi_{\pi}^{p}\left(q^{0}, \vec{q}, \rho\right)=\left(\frac{g_{\pi N N}}{2 M}\right)^{2} \vec{q}^{2} F^{2}(q) \frac{\Pi_{\pi}^{(0)}\left(q^{0}, \vec{q}, \rho\right)}{1-\left(\frac{g_{\pi N N}}{2 M}\right)^{2} g^{\prime}(q) \Pi_{\pi}^{(0)}\left(q^{0}, \vec{q}, \rho\right)},
$$

where $\Pi_{\pi}^{(0)}$ is the sum of the $U_{1 p 1 h}, U_{\Delta h}$ and $U_{2 p 2 h}$ functions, and $g^{\prime}$ is the usual LandauMigdal parameter, which is taken slightly momentum dependent as in Ref. [32].

We have also considered the effects of including form factors at the $\bar{K} N Y$ vertices of Eq. (21). If a monopole form factor with $\Lambda_{K}=1.3 \mathrm{GeV}$ normalized to 1 as $q^{\mu} \rightarrow 0$ is used, the changes observed are very small, at the level of $2 \%$. Since the value of $\Lambda_{K}$ for kaons is not so well known and the changes induced by the form factor are so small, we simply ignore it in the results presented below.

\section{RESULTS}

In this section we discuss the results of our calculations. To facilitate the comparison with other works we will distinguish three types of approximations, all of which include the binding effects on the baryons:

a) Pauli: The blocking of intermediate nucleon states below the Fermi momentum $p_{F}$ is taken into account, but the mesons propagate as in free space. This approach uses the meson-baryon propagator of Eq. (13) for the intermediate states involving nucleons and has been the standard medium effect studied in the literature [9] 11].

b) In-medium kaons: apart from Pauli blocking, the dressed $\bar{K}$ propagator is included in the loops in a self-consistent manner. This approach, which uses Eq. (18) for the intermediate $\bar{K} N$ states, has recently been used in the study of the $K^{-}$properties in the medium [12].

c) In-medium pions and kaons: the dressing of the pions is also considered and, therefore, Eq. (18) is used for the intermediate channels involving both $\bar{K}$ and $\pi$ mesons. This is the main novelty of the present work, apart from considering also the baryon potentials.

To assess the importance of dressing the mesons, we show first their spectral density. In Fig. 1 the spectral density of the $\pi$ mesons in nuclear matter at density $\rho=\rho_{0}$ is shown as a function of energy for several momenta. Note that the scale has been adapted to the two higher momenta such that the structure of the spectral density is more clearly seen. The peak value of the $q=100 \mathrm{MeV} / \mathrm{c}$ spectral function is about $375 \mathrm{GeV}^{-2}$. As the momentum increases the position of the peak moves to higher energies. However, it increasingly deviates 
from the energy $\sqrt{\vec{q}^{2}+m_{\pi}^{2}}$ at which a delta-type spectral density would be located if the dressing of the $\pi$ meson was ignored. The in-medium peak positions are at 158, 179 and $212 \mathrm{MeV}$ for $q=100,200$ and $300 \mathrm{MeV} / \mathrm{c}$, respectively, while the corresponding free values are 172,244 and $331 \mathrm{MeV}$. We also observe, to the left of the peaks, the typical structure of the $1 p 1 h$ excitations, which spreads the pionic strength over a wider energy range.

The spectral funcion of a $K^{-}$meson of zero momentum is shown in Fig. 2 as a function of energy for the three types of approximations used in this work. The solid line shows results for nuclear matter density $\rho=\rho_{0}$, the short-dashed line for $\rho_{0} / 2$ and the long-dashed line for $\rho_{0} / 4$.

The spectral functions shown in the upper panel correspond to the approximation in which only Pauli blocking effects have been considered. For $\rho_{0} / 4$ a two-mode excitation is clearly visible. The left peak corresponds to the $K^{-}$pole branch, appearing at an energy smaller than its mass, $m_{K}$, due to the medium effects which, as we will see, are already attractive at this density. The upper peak corresponds to the $\Lambda(1405)$-hole state, which is located above $m_{K}$ because of the shifting of the $\Lambda(1405)$ resonance to energies above the $K^{-} p$ threshold due to the restrictions on phase space imposed by Pauli blocking. As density increases, the $K^{-}$feels an enhanced attraction and the $K^{-}$pole peak moves to lower energies. At the same time the reduced phase space for the intermediate nucleon states makes the appearance of the resonance less likely. The $\Lambda(1405)$-hole peak moves to higher energies and loses strength, a reflection of the tendency of the $\Lambda(1405)$ to dissolve in the dense nuclear medium. These results are in complete agreement with those obtained by Waas and Weise [11].

When the $\bar{K}$ spectral function displayed in Fig. 2 is insterted in the coupled-channel Bethe-Salpeter equation, the intermediate $\bar{K} N$ states are more spread out over energies. As a consequence, the resulting in-medium interaction, $T_{\text {eff }}$, and the new self-energy calculated from Eq. (19), become softened and distributed over a wider range of energies, affecting in turn the distribution of the $\bar{K}$ spectral strength. After a few iterations of this process, selfconsistency is achieved and the resulting $K^{-}$spectral function is displayed in the middle panel of Fig. 2. The two peak mode is barely visible now. As was noted by Lutz [12], including the in-medium attraction felt by the $K^{-}$through the use of the dressed propagator lowers the threshold for the $\bar{K} N$ states that had been increased by the Pauli blocking on the nucleon. This has a compensatory effect and the resonance barely moves with respect to its free space value. The $K^{-}$pole peak appears at similar or slightly smaller energies, but its width is larger, due to the strength of the intermediate $\bar{K} N$ states being distributed over a wider region of energies. Therefore the $K^{-}$pole and the $\Lambda(1405)$-hole branches merge into one another and can hardly be distinguished.

Finally, the self-consistent $K^{-}$spectral function when the pions in the intermediate loops are also dressed is shown in the bottom panel of Fig. 2. The essential difference with respect to the other two approaches is the fact that the $\pi Y$ channels $(Y=\Lambda$ or $\Sigma)$ start to be operative at lower energies due to the attraction felt by the pions in the medium. As seen by the long-dashed line, even at very small densities one no longer distinguishes the $\Lambda$ (1405)-hole peak from the $K^{-}$pole one. As density increases the attraction felt by the $K^{-}$ is much more moderate and the $K^{-}$pole peak appears at higher energies than in the other two approaches. At normal nuclear matter density a peak starts to develop to the left of that corresponding to the $K^{-}$pole. As will be shown next, this structure is associated to a 
cusp in the imaginary part of the $K^{-}$self-energy at an energy corresponding to the position of the new in-medium $\pi \Sigma$ threshold.

The $K^{-}$self-energy at zero momentum, $\Pi_{K^{-}}\left(q^{0}, 0, \rho\right)$, is displayed in Fig. 3 as a function of the $K^{-}$energy for several densities and for the three approximations discussed here. The graphs on the left show the real part and those on the right the imaginary part. We have seen that the $\bar{K}$ spectral strength is distributed over a wider range of energies and incorporates the attractive medium effects felt by the $\bar{K}$. This is why the self-energy shown in the middle panels (In-medium kaons) is smoother and wider than that displayed on the top (Pauli) ones. Note that $\mathrm{Im} \Pi_{K^{-}}$in the middle panel is non-zero at energies as low as $300 \mathrm{MeV}$. This corresponds to the $N(\Lambda h)$ and $N(\Sigma h)$ many-body channels that are opened at these energies and which are incoporated in the calculation via the $K^{-}$p-wave self-energy. In the lower panels, where the pions are also dressed, we see that $I m \Pi_{K^{-}}$has bigger strength at these low energies. This can be understood in the following way: the $\pi \Sigma$ channel is one of the important building blocks of the $\Lambda(1405)$ strength below the $\bar{K} N$ threshold. When the pions are dressed in the medium they couple strongly to $1 p 1 h$ and $2 p 2 h$ components, so that the $K^{-}$is effectively coupling to intermediate $1 p 1 h \Sigma, 2 p 2 h \Sigma$ states with lower threshold than the $\pi \Sigma$ channel. Actually, the opening of this latter channel on top of the already existing $1 p 1 h \Sigma$ and $2 p 2 h \Sigma$ is visible through a cusp in $\operatorname{Im} \Pi_{K^{-}}$around $q^{0} \simeq 400 \mathrm{MeV}$.

The effect on the $K^{-}$self-energy of the baryon binding potentials is basically a shift in the scale of energies, corresponding to the difference of potentials between the nucleon and the $\Sigma$ hyperon, which amounts to $20 \mathrm{MeV}$ at $\rho=\rho_{0}$.

The scattering properties of a $\bar{K}$ in the nuclear medium will depend on the characteristics of the in-medium $\bar{K} N$ scattering amplitude. In particular, it is interesting to see if the resonant shape of the free $K^{-} p$ scattering amplitude remains when the medium effects are incorporated. Fig. 4 shows the real part (on the left) and imaginary part (on the right) of the $K^{-} p \rightarrow K^{-} p$ scattering amplitude for a total momentum $\left|\vec{p}_{K}+\vec{p}_{N}\right|=0$ as a function of $\sqrt{s}$. Results are shown for several densities and for the three types of approximations. On the top panels we can see how, as density increases, the Pauli blocking effects merely shift the resonance to higher energies, hence changing from being $27 \mathrm{MeV}$ below the $K^{-} p$ threshold to being above it from a certain density on. Note, however, that the resonance still appears, for each density, below the new threshold imposed by the Pauli principle, i.e. $m_{K}+M+p_{F}^{2} / 2 M$. On the other hand, the resonance shape remains pretty much unaltered. When the dressing of the $\bar{K}$ meson is also incorporated, we observe how the resonant shape smears out quite fast with increasing density. Moreover, the resonance does not move up to higher energies but stays close to the free space value due to a cancellation between the repulsive Pauli blocking effects and the attractive medium effects on the $\bar{K}$. These results are in qualitative agreement with the self-consistent calculation of Lutz [12, although we seem to observe a faster dilution of the resonance with the medium density. Finally, when the in-medium dressing of the pions are also included we obtain a quite similar pattern. The resonance dissolves very fast although it shows a tendency to move to higher energies.

The isospin averaged in-medium scattering length defined as

$$
a_{\mathrm{eff}}=-\frac{1}{4 \pi} \frac{M}{m_{K}+<E_{N}>} \frac{\prod_{\bar{K}}\left(m_{K}, \vec{q}=0, \rho\right)}{\rho}
$$


with $<E_{N}>=M+U_{N}(\rho)+\frac{3}{5}\left(\frac{p_{F}^{2}}{2 M}\right)$, is shown if Fig. 5. The change of Re $a_{\text {eff }}$ from negative to positive values indicates the transition from a repulsive interaction in free space to an attractive interaction in the medium. As shown by the dotted line, this transition happens at a density of about $\rho \sim 0.1 \rho_{0}$ when only Pauli effects are considered, in agreement with what was found in Ref. [10]. However, this transition occurs at even lower densities $\left(\rho \sim 0.04 \rho_{0}\right)$ when one considers the dressing of the mesons in the description, as seen from the dashed (In-medium kaons) and full (In-medium pions and kaons) lines. The deviations from the approach including only Pauli blocking or those dressing the mesons are quite appreaciable over a wide range of densities.

The effective $K^{-}$mass is shown in Fig. 6 as a function of $\rho / \rho_{0}$ for the different approximations. Note that the effective mass, defined as the solution of Eq. (26) for $\vec{q}=0$, gives the position of the $K^{-}$pole peak in the spectral function assuming a weak energy dependence of $\operatorname{Im} \Pi_{K^{-}}\left(q^{0}, 0, \rho\right)$. In addition, one can only think of the $K^{-}$as a quasi-meson moving with the modified $m_{K^{-}}^{*}$ mass if the spectral function shows a clear and narrow enough $K^{-}$ pole peak, which happens when $\operatorname{Im} \Pi_{K^{-}}\left(q^{0}, 0, \rho\right)$ is sufficiently small. By looking at the $K^{-}$ spectral function of Fig. 2 one sees that these conditions are not always fulfilled, especially when the dressing of the mesons is incorporated. Keeping this in mind, we can still analyze the effective mass as a simplified way to assess how important is the attraction felt by the $K^{-}$meson in the medium. Morover, this analysis will allow to compare our results with those obtained by other calculations. As can be seen in the zoomed area on the top right of the figure, the in-medium $K^{-}$mass increases slightly from the free value but quickly gains attraction as density increases. The transition from repulsion to attraction occurs around $0.1 \rho_{0}$ for the Pauli approximation (dotted line) while the two other approximations that incorporate the dressing of the $\bar{K}$ (dashed line) and also that of the pion (full line) show attraction already at around $0.04 \rho_{0}$. However, the density dependence is stronger with the Pauli approximation which gives rise to a lower value of $m_{K^{-}}^{*}$ from $0.11 \rho_{0}$ onwards. It turns out that, in spite of the essentially different shapes of the $K^{-}$spectral function, the position of the $K^{-}$pole is very similar for the Pauli and the In-medium kaons approximations for densities higher than $\rho_{0}$. Consequently, the calculated effective masses decrease similarly. However, when the dressing of the pions is also included, the attraction is substantially more moderate and the effective mass seems to level off at high densities around the value achieved already at $\rho_{0}$ of $m_{K^{-}}^{*}=0.9 m_{K}=445 \mathrm{MeV}$. This would correspond to a $K^{-}$optical potential of $U_{K^{-}}=\operatorname{Re} \Pi_{K^{-}}\left(m_{K^{-}}^{*}, \overrightarrow{0}, \rho_{0}\right) /\left(2 m_{K^{-}}^{*}\right) \sim-50 \mathrm{MeV}$. The Pauli and In-medium kaons approximations give rise to a a more attractive value of the effective mass at $\rho_{0}$, namely $m_{K^{-}}^{*}=415 \mathrm{MeV}$ which corresponds to a $U_{K^{-}}=-86 \mathrm{MeV}$. It is worth mentioning that all the calculations, either microscopic [10,11] or based on mean field theories [4, 14, 16] predict effective masses at $\rho_{0}$ which range between $375 \mathrm{MeV}$ and $425 \mathrm{MeV}$. The corresponding $K^{-}$potential at the center of the nucleus would then be in between -140 and -75 $\mathrm{MeV}$. Therefore, no theory is able to explain the large attraction of -200 obtained from the best available fit to kaonic atom data [5]. However, we should warn that the data of $K^{-}$atoms are only sensitive to the $K^{-}$self-energy at the low effective densities felt by the kaons that explore basically the nuclear surface. The values obtained by the fit in Ref. [5] at $\rho=\rho_{0}$ are an extrapolation of a preassumed functional dependence on $\rho$. It is possible to obtain equally good fits with other functionals that give the same value for the low densities 
explored by the kaons but different extrapolations at $\rho=\rho_{0}$. In fact, quite good fits to the $K^{-}$atoms data are also obtained with the Batty potential of Ref. [33] which is of the order of $-50 \rho / \rho_{0} \mathrm{MeV}$. We also note that the Brueckner-type calculations of Ref. [13] obtained a shallow $K^{-}$-nucleus potential, of the order of $-40 \mathrm{MeV}$ at the center of ${ }^{12} \mathrm{C}$, and predicted reasonably well the data available at that time.

With the values obtained here for $m_{K^{-}}^{*}$ and similar ones that we obtain for neutron matter the phenomenon of $K^{-}$condensation appears very unlikely, since there is not enough attraction for the $K^{-}$energy to become smaller than the electron chemical potential in beta stable neutron star matter.

The implications of our results for the in-medium $K^{-}$self-energy on kaonic atoms is currently being analyzed [34] and gives results compatible with the existing kaonic atom data. That analysis, based on a local density approximation, requires the knowledge of the optical potential, defined as

$$
U_{K^{-}}(\omega, \rho)=\frac{\Pi_{K^{-}}\left(\omega, q_{\mathrm{on}}, \rho\right)}{2 \omega},
$$

where $q_{\text {on }}$ is the on-shell momentum value that fulfills Eq. (26) for a given value of $\omega$. The real and imaginary parts of the optical potential for the In-medium pions and kaons approximation are shown in Fig. 07 for three energy values: $m_{K}-45 \mathrm{MeV}$ (dotted line), $m_{K}$ (solid line) and $m_{K}+25 \mathrm{MeV}$ (dashed line). The self-energy shown by the solid lines is the relevant one for studies of low lying $K^{-}$atomic states, while the results displayed by the dotted line would be the ones to use for very deeply bound states in the $\mathrm{Pb}$ region. The dashed lines show an example of the $K^{-}$optical potential at positive energies and would be the one to consider to treat the $K^{-}$distortions in $K^{-}$-nucleus scattering reactions around $25 \mathrm{MeV}$. While the real part of the optical potential becomes less attractive with increasing energy, the imaginary part does not show a too strong energy dependence in the range of energies explored. This behavior can be easily inferred from the $K^{-}$self-energies shown at the bottom of Fig. 3 .

\section{CONCLUSIONS}

In this work we have studied the $K^{-}$properties in nuclear matter using a chiral unitary approach for the $K^{-} N$ interaction in s-wave that incorporates the medium effects microscopically.

Pauli blocking acting on the intermediate nucleon states modifies the $K^{-} N$ interaction from that in free space and gives rise to a $K^{-}$spectral function that shows two distinctive peaks at very low density. The lower one corresponds to the the position of the new $K^{-}$pole, located below the $K^{-}$mass from already quite low densities on, which indicates that the $K^{-}$ feels an attraction in the medium. The higher peak is associated to the in-medium $\Lambda(1405)$ resonance that appears above the free $K^{-} p$ threshold due to the repulsive effect induced by blocking the intermediate nucleon states below the Fermi momentum. As density increases, the $K^{-}$feels more attraction and the $\Lambda(1405)$ keeps moving to higher energies.

When the $K^{-}$self-energy is incorporated self-consistently into the scheme, there is a compensation between the attraction felt by the $K^{-}$and the repulsive Pauli blocking shift. As a result, the resonance appears at a similar location as that in free space. However, 
its width is much larger and it tends to dissolve with increasing density due to a weaker in-medium $K^{-} N$ interaction which now is determined from dressed $\bar{K}$ propagators which are more spread out over energies.

In the present work we also include the dressing of the pions in the intermediate $\pi \Lambda$, $\pi \Sigma$ states to which the $\bar{K} N$ system can couple. We use a pion self-energy that includes the coupling to $1 p 1 h, \Delta h$ and $2 p 2 h$ excitations modified by the effect of short-range $N N$ correlations. The fact that now the pions also have a spreading width makes the in-medium $\bar{K} N$ interaction even smoother. The $K^{-}$feels less attraction and the $\Lambda(1405)$ resonance, which is shifted slighty upwards from its free space position, dissolves even faster with density.

Our approach gives rise to a $K^{-}$self-energy at normal nuclear density which has about half the attraction of that obtained with other theories and approximation schemes and

would make the phenomenon of kaon condensation very unlikely. The self-energy obtained is, however, compatible with the existing data on kaonic atoms.

\section{ACKNOWLEDGMENTS}

This work is partially supported by DGICYT contract numbers PB95-1249 and PB960753, and by the EEC-TMR Program under contract No. CT98-0169. 


\section{REFERENCES}

[1] D.B. Kaplan and A.E. Nelson, Phys. Lett. B175 (1986) 57

[2] R. Barth et al., Phys. Rev. Lett. 78 (1997) 4007

[3] W. Cassing, E.L. Bratkovskaya, U. Mosel, S. Teis and A. Sibirtsev, Nucl. Phys. A614 (1997) 415; E.L. Bratkovskaya, W. Cassing and U. Mosel, Nucl. Phys. A622 (1997) 593

[4] G.Q. Li, C.-H. Lee and G.Brown, Nucl. Phys. A625 (1997) 372; ibid, Phys. Rev. Lett. 79 (1997) 5214

[5] E. Friedman, A. Gal, C.J. Batty, Nucl. Phys. A579 (1994) 518

[6] C.-H. Lee, G.E. Brown, D.P. Min and M. Rho, Nucl. Phys. A385 (1995) 481; C.-H. Lee, Phys. Rep. 275 (1996) 255

[7] N. Kaiser, P.B. Siegel and W. Weise, Nucl. Phys. A594 (1995) 325; N. Kaiser, T. Waas and W. Weise, Nucl. Phys. A612 (1997) 297

[8] E. Oset and A. Ramos, Nucl. Phys. A635 (1998) 99

[9] V. Koch, Phys. Lett. B337 (1994) 7

[10] T. Waas, N. Kaiser and W. Weise, Phys. Lett. B365 (1996) 12; ibid. B379 (1996) 34

[11] T. Waas and W. Weise, Nucl. Phys. A625 (1997) 287

[12] M. Lutz, Phys. Lett. B426 (1998) 12

[13] M. Alberg, E.M. Henley and L. Wilets, Ann. Phys. 96 (1976) 43

[14] G. Mao, P. Papazoglou, S. Hofmann, S. Schramm, H. Stöcker and W. Greiner, Phys. Rev. C, in press. nucl-th/9806068

[15] J. Schaffner and I.N. Mishustin, Phys. Rev. C53 (1996) 1416;

J. Schaffner-Bielich, I.N. Mishustin and J. Bondorf, Nucl. Phys. A625 (1997) 325

[16] K. Tsushima, K. Saito, A.W. Thomas and S.V. Wright, Phys. Lett. B429 (1998) 239

[17] J. Gasser and H. Leutwyler, Nucl. Phys. B250 (1985) 465

[18] A. Pich, Rep. Prog. Phys. 58 (1995) 563

[19] G. Ecker, Prog. Part. Nucl. Phys. 35 (1995) 1

[20] V. Bernard, N. Kaiser and U.G. Meissner, Int. J. Mod. Phys. E4 (1995) 193

[21] J.A. Oller, E. Oset and J.R. Peláez, Phys. Rev. Lett. 80 (1998) 2452

[22] D.N. Tovee et al., Nucl. Phys. B33 (1971) 493

[23] R.J. Nowak et al., Nucl. Phys. B139 (1978) 61

[24] A.D. Martin, Nucl. Phys. B179 (1981) 33

[25] M. Iwasaki et al., Phys. Rev. Lett. 78 (1997) 3067

[26] J.A. Nacher, E. Oset, H. Toki and A. Ramos, submitted to Phys. Lett. B

[27] H. Bandō, T. Motoba and J. Žofka, Int. J. Mod. Phys. A5 (1990) 4021

[28] T. Nagae et al., Phys. Rev. Lett. 80 (1998) 1605

[29] C.J. Batty et al, Phys. Lett. 74B (1978) 27, ibid. 87B (1979) 324

[30] E. Oset, P. Fernández de Córdoba, L.L. Salcedo and R. Brockmann, Phys. Reports 188 (1990) 79

[31] O. Meirav, E. Friedman, R.R. Johnson, R. Olszewski and P. Weber, Phys. Rev. C40 (1989) 843

[32] A. Ramos, E. Oset and L.L. Salcedo, Phys. Rev. C50 (1994) 2314

[33] C.J. Batty, Nucl. Phys. A372 (1981) 418

[34] S. Hirenzaki, Y. Okumura, H. Toki, E. Oset and A. Ramos, in preparation. 


\section{TABLES}

TABLE I. $K^{-} p$ threshold ratios and $K^{-} N$ scattering lengths

\begin{tabular}{lcc}
\hline \hline & This work & Exp. \\
\hline$\gamma=\frac{\Gamma\left(K^{-} p \rightarrow \pi^{+} \Sigma^{-}\right)}{\Gamma\left(K^{-} p \rightarrow \pi^{-} \Sigma^{+}\right)}$ & 2.32 & $2.36 \pm 0.04$ [22,23] \\
$R_{c}=\frac{\Gamma\left(K^{-} p \rightarrow \text { charged particles }\right)}{\Gamma\left(K^{-} p \rightarrow \text { all }\right)}$ & 0.627 & $0.664 \pm 0.011$ [22, 23] \\
& & \\
$R_{n}=\frac{\Gamma\left(K^{-} p \rightarrow \pi^{0} \Lambda\right)}{\Gamma\left(K^{-} p \rightarrow \text { neutral states }\right)}$ & 0.213 & $0.189 \pm 0.015$ [22, 23] \\
$a_{K^{-} p}(\mathrm{fm})$ & $-1.00+i 0.94$ & $-0.67+i 0.64$ [24] 24] \\
& & $-0.98(f r o m \operatorname{Re}(a))$ 24] \\
$a_{K^{-} n}(\mathrm{fm})$ & $0.53+i 0.62$ & $0.37+i 0.60$ [24] \\
\hline \hline
\end{tabular}

TABLE II. SU(3) coupling constants defined in Eq. (20)

\begin{tabular}{c|cc|c|cc|}
\hline \hline & & $K^{-}$ & & \multicolumn{2}{c|}{$\bar{K}^{0}$} \\
& $\alpha_{M B B^{\prime}}$ & $\beta_{M B B^{\prime}}$ & & $\alpha_{M B B^{\prime}}$ & $\beta_{M B B^{\prime}}$ \\
\hline$p \Lambda$ & $-\frac{2}{\sqrt{3}}$ & $\frac{1}{\sqrt{3}}$ & $n \Lambda$ & $-\frac{2}{\sqrt{3}}$ & $\frac{1}{\sqrt{3}}$ \\
$p \Sigma^{0}$ & 0 & 1 & $n \Sigma^{0}$ & 0 & -1 \\
$n \Sigma^{-}$ & 0 & $\sqrt{2}$ & $p \Sigma^{+}$ & 0 & $\sqrt{2}$ \\
\hline \hline
\end{tabular}




\section{FIGURES}

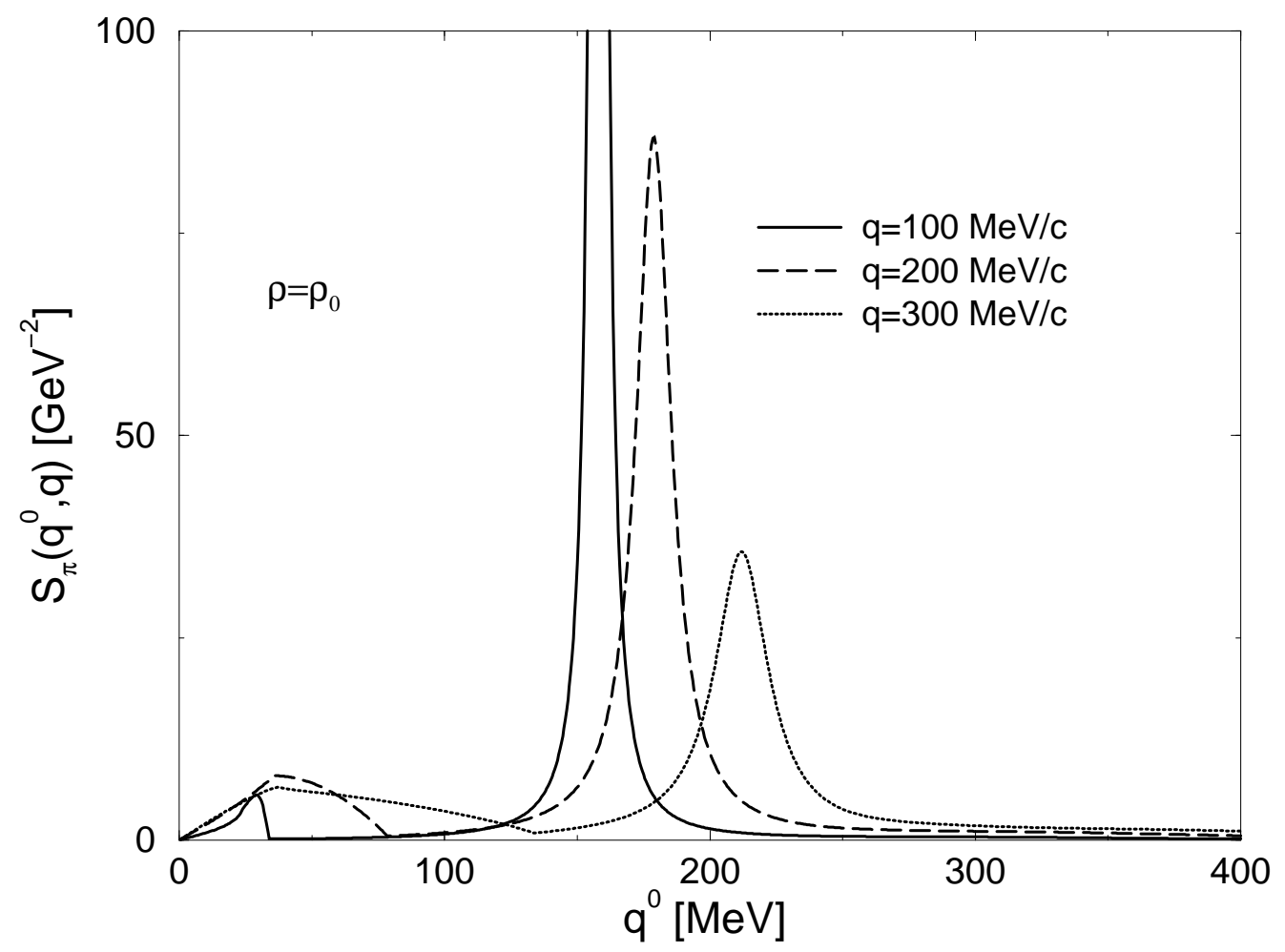

FIG. 1. Pion spectral density at normal nuclear matter density as a function of energy for several pion momenta: $q=100 \mathrm{MeV} / \mathrm{c}$ (solid line), $q=200 \mathrm{MeV} / \mathrm{c}$ (dashed line) and $q=300$ $\mathrm{MeV} / \mathrm{c}$ (dotted line). 


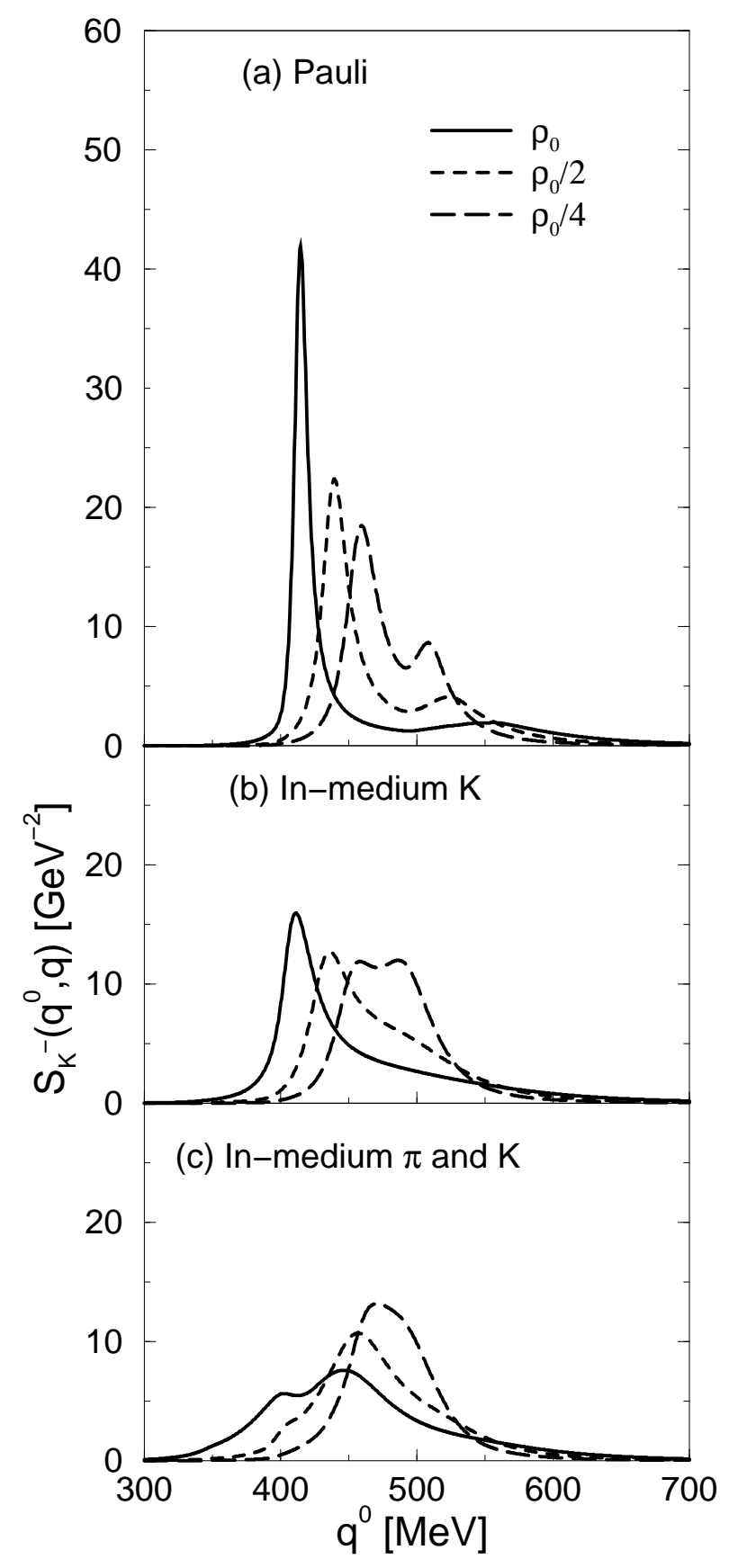

FIG. 2. $K^{-}$spectral density for zero momentum as a function of energy at several densities: $\rho_{0}$ (solid line), $\rho_{0} / 2$ (short-dashed line) and $\rho_{0} / 4$ (long-dashed line). Results are shown for the three approximations discussed in the text: a) Pauli (top panels), b) In-medium kaons (middle panels) and c) In-medium pions and kaons (bottom panels). 


\section{$\operatorname{Re} \Pi_{K}{ }^{-}\left[\mathrm{GeV}^{2}\right] \quad \operatorname{Im} \Pi_{\mathrm{K}}^{-}\left[\mathrm{GeV}^{2}\right]$}

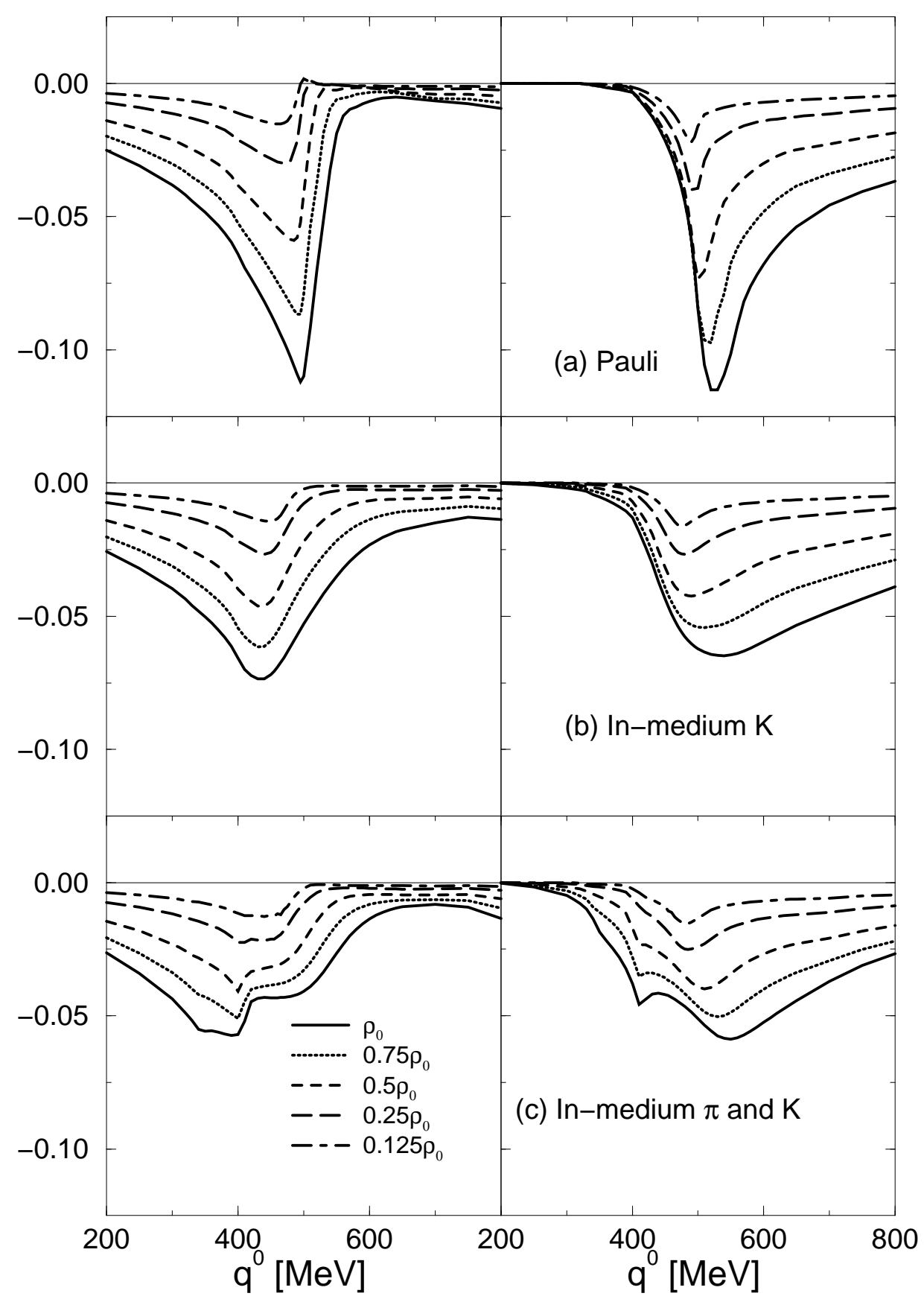

FIG. 3. Real (left) and imaginary (right) parts of the zero momentum $K^{-}$self-energy as a function of energy, for several nuclear matter densities. Results are shown for the three approximations discussed in the text: a) Pauli (top panels), b)In-medium kaons (middle panels) and c) In-medium pions and kaons (bottom panels). 


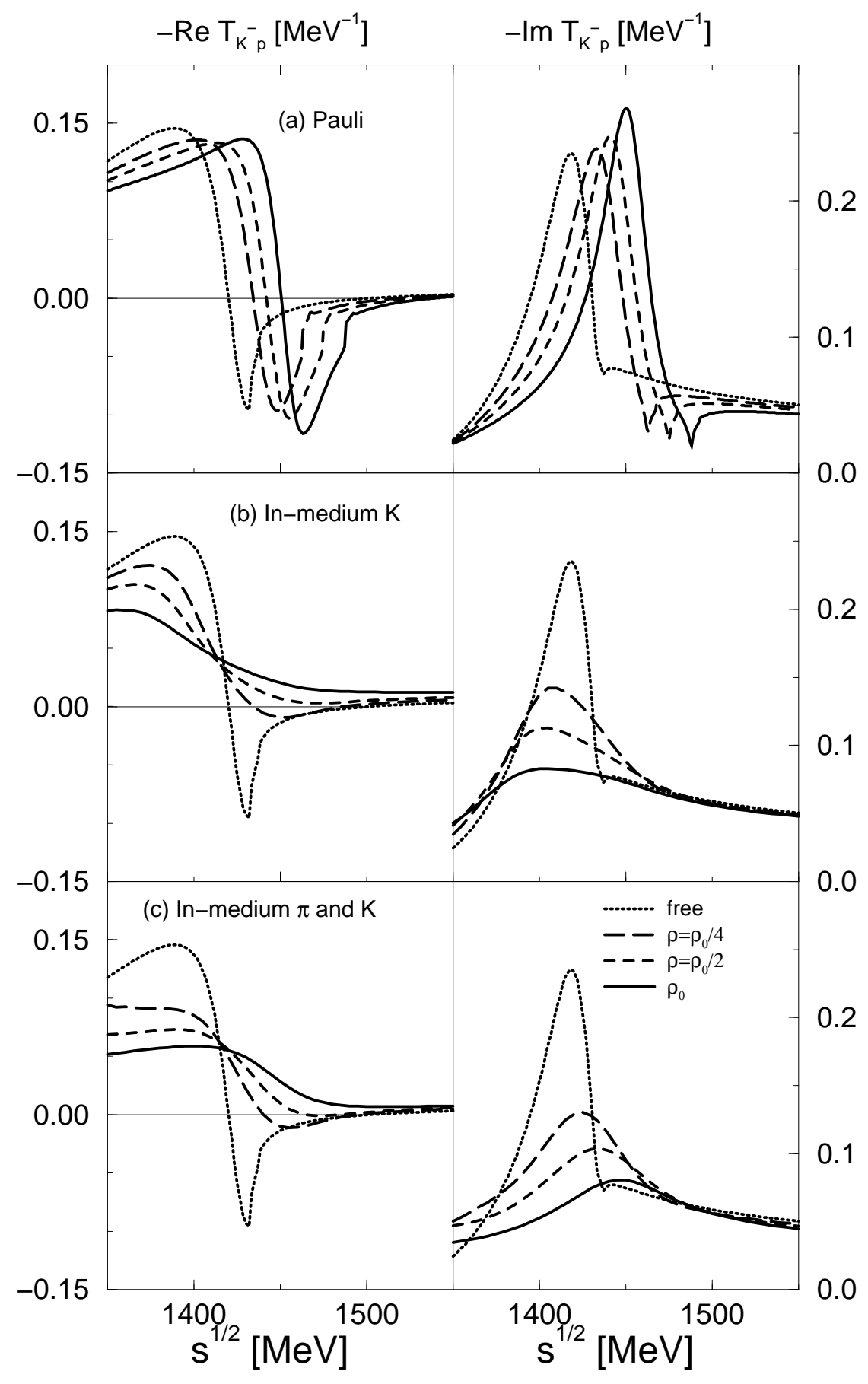

FIG. 4. Real (left) and imaginary (right) parts of the in-medium $K^{-} p$ scattering amplitude as a function of the invariant energy $\sqrt{s}$ for $\left|\vec{p}_{K^{-}}+\vec{p}_{N}\right|=0$ and several densities. Results are shown for the three approximations discussed in the text: a) Pauli (top panels), b) In-medium kaons (middle panels) and c) In-medium pions and kaons (bottom panels). 


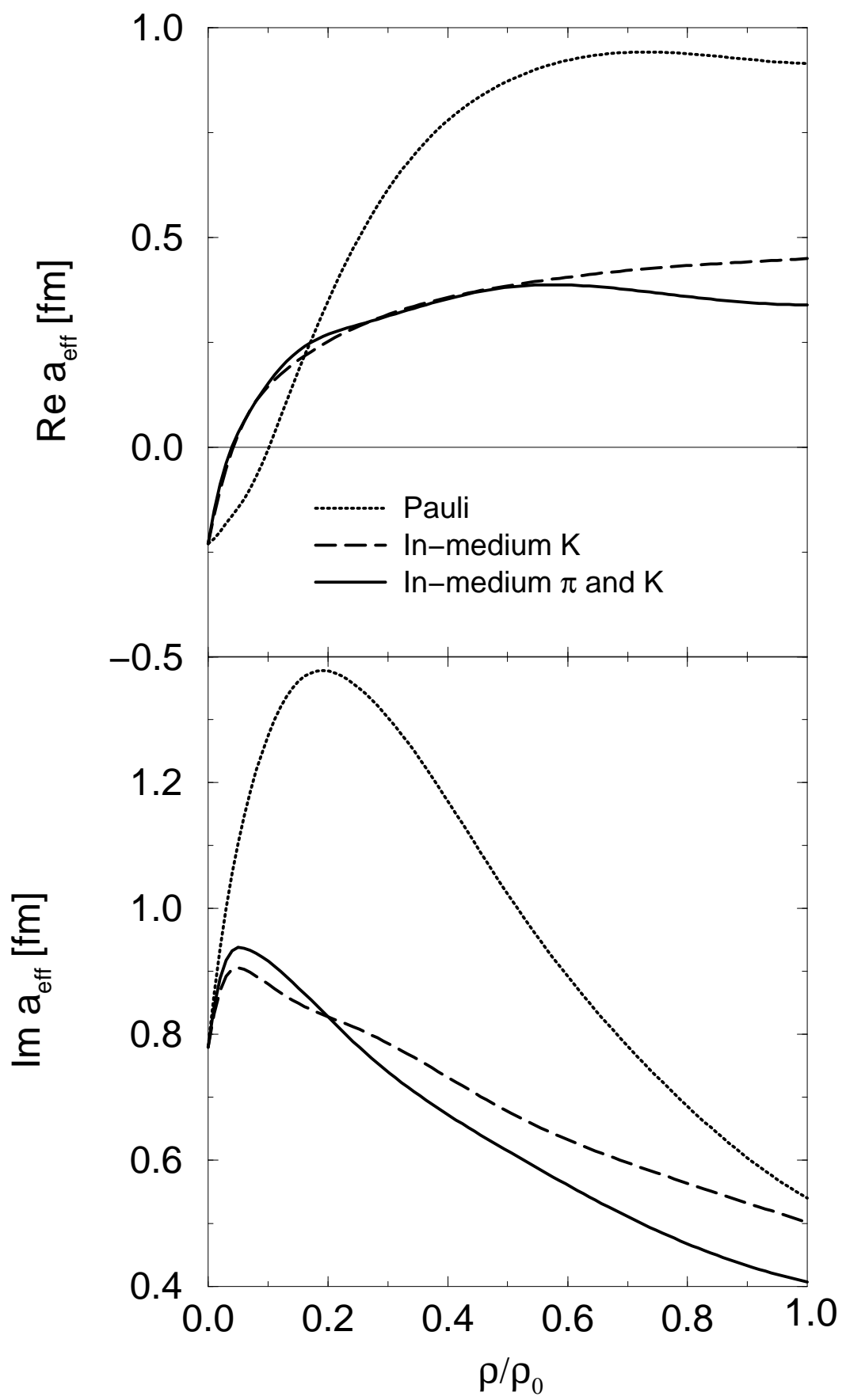

FIG. 5. Real (top) and imaginary (bottom) parts of the in-medium $K^{-} N$ scattering length as a function of density for the three approximations discussed in the text: Pauli (dotted lines), In-medium kaons (dashed lines) and In-medium pions and kaons (solid lines). 


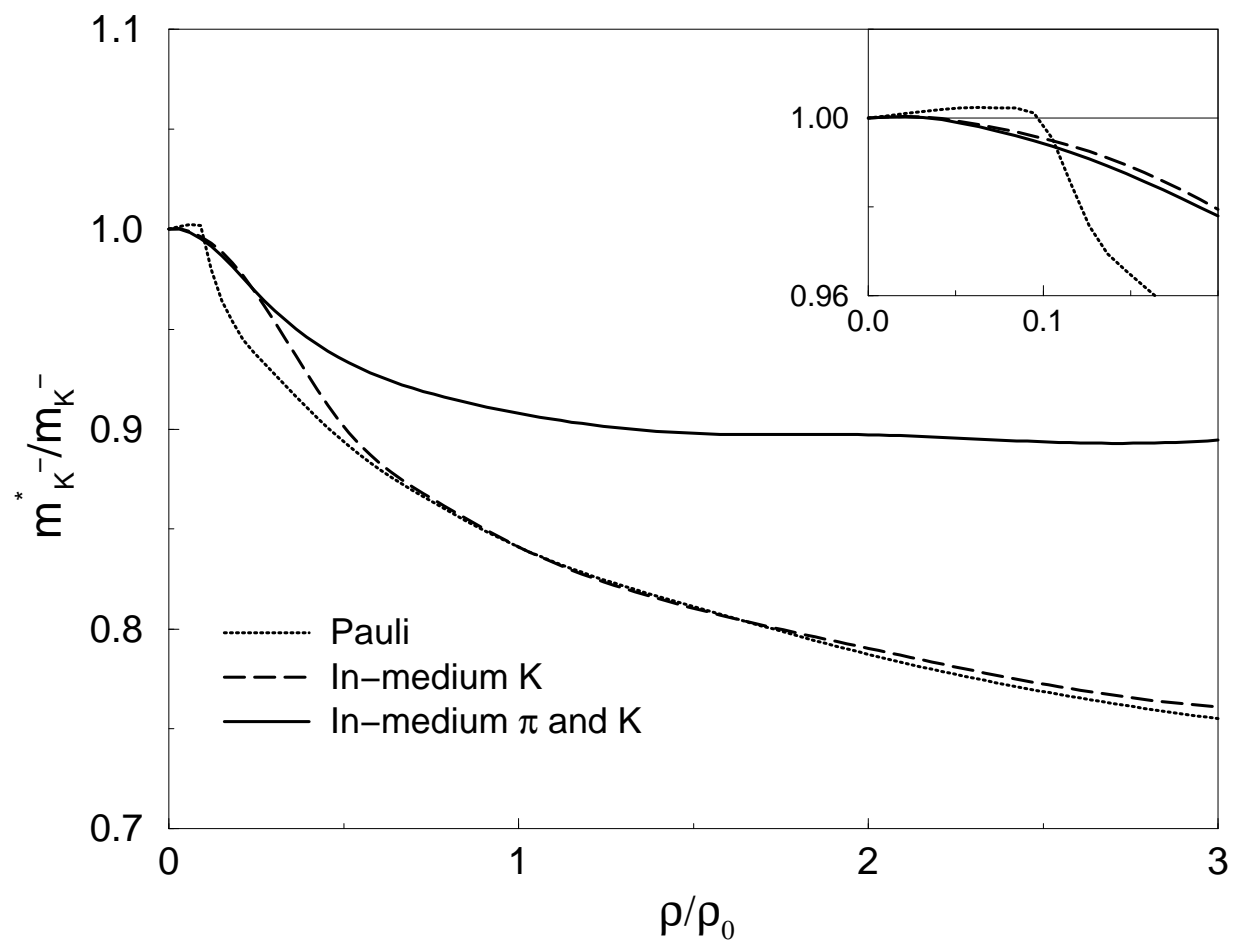

FIG. 6. Effective mass of the $K^{-}$meson in nuclear matter as a function of density for the three approximations discussed in the text: Pauli (dotted lines), In-medium kaons (dashed lines) and In-medium pions and kaons (solid lines). 


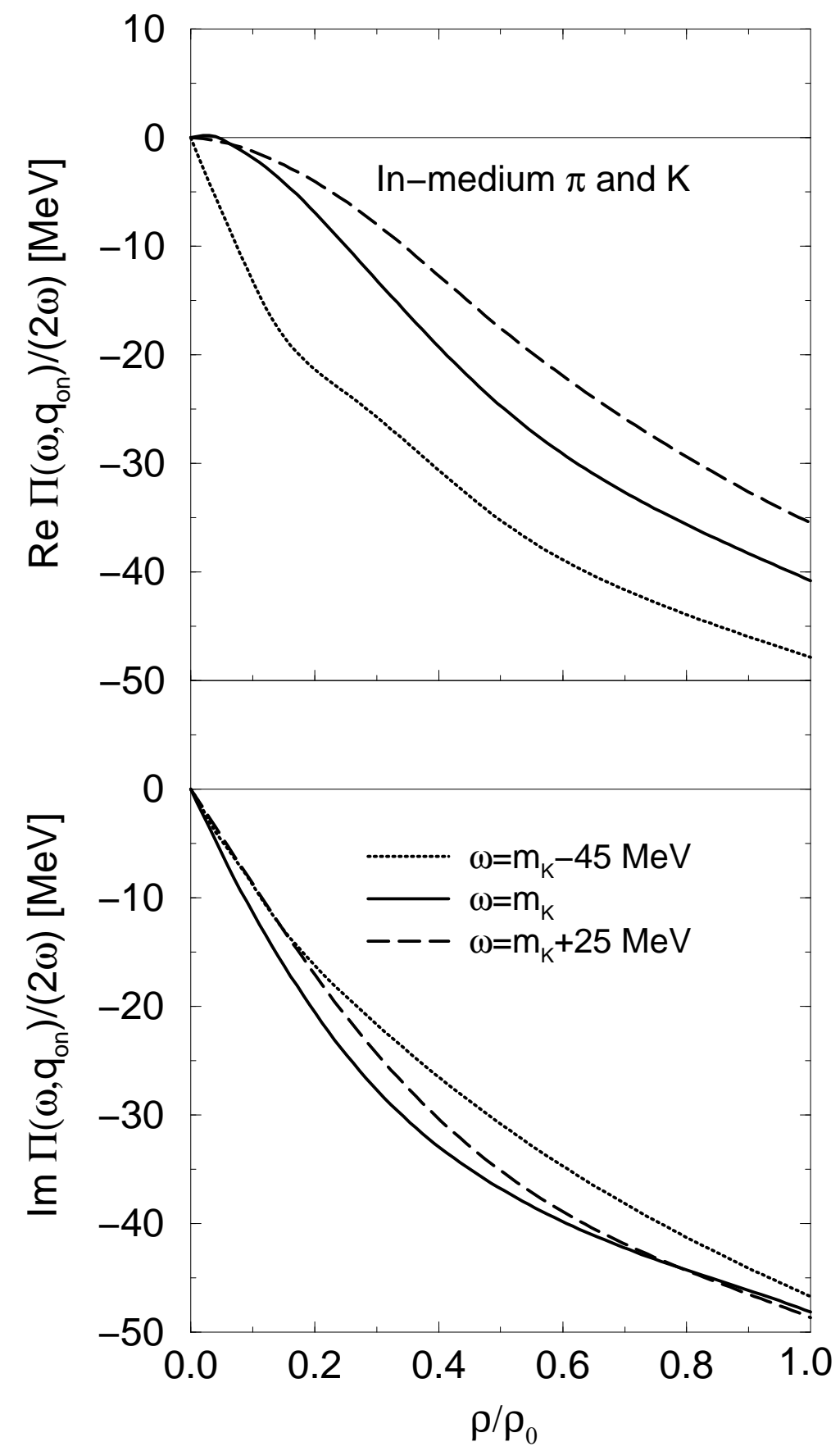

FIG. 7. Real (top) and imaginary (bottom) parts of the $K^{-}$optical potential as a function of density obtained from the In-medium pions and kaons approximation. Results are shown for three different $K^{-}$energies: $\omega=m_{K}-45 \mathrm{MeV}$ (dotted lines), $\omega=m_{K}$ (solid lines) and $\omega=m_{K}+20$ $\mathrm{MeV}$ (dashed lines). 